\title{
Vision of a pictorial hand modulates visual-tactile interactions
}

\author{
YUKA IGARASHI \\ Tokyo Metropolitan University, Tokyo, Japan \\ NORIMICHI KITAGAWA \\ Nihon University, Tokyo, Japan \\ and \\ SHIGERU ICHIHARA \\ Tokyo Metropolitan University, Tokyo, Japan
}

\begin{abstract}
The participants in this study discriminated the position of tactile target stimuli presented at the tip or the base of the forefinger of one of the participants' hands, while ignoring visual distractor stimuli. The visual distractor stimuli were presented from two circles on a display aligned with the tactile targets in Experiment 1 or orthogonal to them in Experiment 2. Tactile discrimination performance was slower and less accurate when the visual distractor stimuli were presented from incongruent locations relative to the tactile target stimuli (e.g., tactile target at the base of the finger with top visual distractor) highlighting a cross-modal congruency effect. We examined whether the presence and orientation of a simple line drawing of a hand, which was superimposed on the visual distractor stimuli, would modulate the cross-modal congruency effects. When the tactile targets and the visual distractors were spatially aligned, the modulatory effects of the hand picture were small (Experiment 1). However, when they were spatially misaligned, the effects were much larger, and the direction of the cross-modal congruency effects changed in accordance with the orientation of the picture of the hand, as if the hand picture corresponded to the participants' own stimulated hand (Experiment 2). The results suggest that the two-dimensional picture of a hand can modulate processes maintaining our internal body representation. We also observed that the cross-modal congruency effects were influenced by the postures of the stimulated and the responding hands. These results reveal the complex nature of spatial interactions among vision, touch, and proprioception.
\end{abstract}

The perception of our own body is essential for our daily life. When we interact with the outside world, we need to know where our body is, what space it occupies, and what posture it assumes. Recent studies have shown that vision plays an important role in perceiving the body through interactions with other senses, such as touch and proprioception. Our internal representation of the body and peripersonal space is constructed and updated through the integration of these different senses (see Làdavas, 2002, and Maravita, Spence, \& Driver, 2003, for reviews). Recent research in this area has been carried out in many different neuroscientific disciplines, such as psychological studies of normal participants (Botvinick \& Cohen, 1998;

N.K. and S.I. were supported by Grants in Aid for Scientific Research, Ministry of Education, Science, and Culture, Japan to S.I. (No. 15330157). N.K. was also supported by a JSPS Research Fellowship for Young Scientists. We thank Nicholas Holmes, Charles Spence, and Hiromi Akutsu for helpful comments on this study. Correspondence concerning this article should be addressed either to Y. Igarashi, Department of Psychology, Tokyo Metropolitan University, 1-1, Minami-osawa, Hachioji, Tokyo 192-0397, Japan (e-mail: igarasi-yuka@c.metro-u.ac.jp) or to N. Kitagawa, Human Information System Laboratory, Kanazawa Institute of Technology, 3-1 Yatsuhako, Matto, Ishikawa 924-0838, Japan (e-mail: kitagawa@kanazawa-it.ac.jp).
Pavani, Spence, \& Driver, 2000; Spence, Pavani, \& Driver, 2004), neuropsychological studies of brain-damaged patients (di Pellegrino, Làdavas, \& Farnè, 1997; Farnè, Pavani, Meneghello, \& Làdavas, 2000; Làdavas, Farnè, Zeloni, \& di Pellegrino, 2000; Spence, Kingstone, Shore, \& Gazzaniga, 2001), neurophysiological studies of monkeys (Graziano, 1999; Graziano, Cooke, \& Taylor, 2000), and functional neuroimaging studies of normal human participants (Lloyd, Shore, Spence, \& Calvert, 2003).

It has been reported that not only direct viewing of own body parts, but even indirect viewing of them can influence visual-tactile interactions. For example, seeing one's own hands in a mirror reflection modulates visuotactile discrimination performance (Maravita, Spence, Sergent, \& Driver, 2002; Soto-Faraco, Ronald, \& Spence, in press). An interesting aspect of seeing our own hands in a mirror is that we receive both tactile and proprioceptive information regarding our hands in personal space, whereas the visual information is seen at a distance (often in extrapersonal space). Similarly, it has been reported that seeing one's own body parts in a video monitor can also affect tactile performance for stimuli presented at the body parts seen (Tipper et al., 1998; Tipper et al., 2001). More recently, Pavani and Castiello (2004) reported that 
even a shadow of one's own hand could modulate visualtactile interactions. Taken together, these studies suggest that visual cues providing information regarding our own body play an important role in maintaining the internal body representation, even when they are presented on a two-dimensional plane and when presented from different locations in touch and proprioception.

One interesting question to emerge from the results mentioned above is whether a two-dimensional simple drawing, or picture, of a body part can influence interactions between vision and touch. For example, when playing a video game or manipulating a computer mouse, we receive visual information and tactile stimulation from different locations, and the visual object that we manipulate is usually just an image on a screen. Once we get used to the manipulation, we rarely notice the discrepancy between the visual and the tactile feedback, and we may sometimes feel as if the two-dimensional object on a display is a part of our own body. In this study, we used the cross-modal congruency task to examine whether seeing a picture of a hand modulates visual-tactile interactions.

The cross-modal congruency task has proven to be a useful paradigm for examining various aspects of visualtactile interactions (see, e.g., Maravita et al., 2003, for a review; Spence et al., 2004). In this task, two tactile stimulators are positioned to the thumb and forefinger of both hands. On each trial in a typical study, a tactile target stimulus and a visual distractor stimulus are presented randomly and independently at one of four locations. The participant's task is to judge the elevation of the vibrotactile target (upper or lower, regardless of which hand is stimulated) as rapidly and accurately as possible, while ignoring any visual distractors that may be presented. The speed and accuracy of tactile discrimination performance is typically worse when the stimuli are incongruent (e.g., a lower tactile target with an upper visual distractor) than when they are congruent. The difference in performance between incongruent- and congruent-distractor trials (i.e., the cross-modal congruency effect) provides a measure of the extent to which people can selectively attend to one sensory modality and ignore irrelevant stimuli from another sensory modality. Recently, spatial variations in the magnitude of the cross-modal congruency effect have been used to investigate the possible nature of the representation of the body when we see our own limbs in a mirror reflection (Maravita, Spence, Sergent, \& Driver, 2002; Soto-Faraco et al., in press) and when we see even a shadow of our hands (Pavani \& Castiello, 2004). Therefore, the cross-modal congruency effect would provide an ideal paradigm for exploring the effect of a hand picture on visual-tactile interactions.

We investigated whether the magnitude of the crossmodal congruency effect would be affected by the presence of a picture of a hand and the orientation of that picture. Tactile target stimuli were presented at either the tip or the base of the forefinger of the participant's left hand in Experiment 1 or of the left or right hand in Experiment 2 . The participants' task was to discriminate the po- sition of the tactile target stimuli (the tip or base) while ignoring visual distractor stimuli. Visual distractor stimuli were presented from two small circles on a display aligned with the tactile targets in Experiment 1 and orthogonal to them in Experiment 2. A picture of a hand was superimposed on the visual distractors so that they were positioned at the tip and base of the forefinger of the hand picture. The hand picture was presented in two orientation conditions (i.e., upward or downward orientation in Experiment 1 and leftward or rightward orientation in Experiment 2). If the picture of the hand has any modulatory effect on visual-tactile interactions, the presence of the picture would be expected to increase the magnitude of the cross-modal congruency effect, as compared with when no picture was presented, and the direction of the cross-modal congruency effect (i.e., which visual distractor leads to slower and less accurate tactile discriminations at the tip or the base of the forefinger) would change along with the orientation of the hand picture.

Although we concentrated on how visual distractors and tactile targets are spatially correlated with each other, it has been reported that task performance also depends on the mapping between stimulus and response arrays. This effect is referred to by the term stimulus-response (S-R) compatibility (see Cho \& Proctor, 2003, for a recent review). Responses are faster and more accurate when stimulus and response arrays are spatially compatible than when they are incompatible. For example, people can respond more rapidly and accurately when they respond to a left stimulus with a left response key and a right stimulus with a right response key than with the opposite keys (e.g., Fitts \& Deininger, 1954). S-R compatibility effects are also observed even when stimulus and response arrays are orthogonal to each other (e.g., two visual stimuli in a vertical array and two response buttons in a horizontal array). Two types of orthogonal S-R compatibility effects have been reported (see Cho \& Proctor, 2003; Lippa \& Adam, 2001). The first type is an overall advantage of up-right/down-left mapping. That is, when the top stimulus is paired with the right response and the bottom stimulus is paired with the left response (i.e., up-right/ down-left mapping), people respond more rapidly than when the stimuli are assigned to the opposite responses (i.e., up-left/down-right mapping; e.g., Weeks \& Proctor, 1990). In the second type of orthogonal S-R compatibility effect, S-R mapping preference varies with the responding hand, its posture, and the spatial relationship between stimulus and response arrays (Bauer \& Miller, 1982; Lippa, 1996; Lippa \& Adam, 2001).

These S-R compatibility results have some implications for the present interest in how visual distractors and tactile targets are paired with each other, especially in the case of Experiment 2, where the visual distractors and tactile targets were positioned orthogonally to each other. In addition, in most previous studies concerning S-R compatibility effects, the relationships between task-relevant stimuli and responses to them have been examined. It would, therefore, be interesting to investigate whether S-R 
compatibility effects are also observed for task-irrelevant stimuli, such as the visual distractors in the cross-modal congruency task. For this reason, the response button array was always positioned in a plane orthogonal to the visual distractors in the present experiments.

\section{EXPERIMENT 1}

\section{Method}

Participants. Eight participants (4 males and 4 females; mean age, 21.8 years; range, 20-25 years) took part in the experiment. All the participants had normal touch and normal or corrected-tonormal visual acuity. All were right-handed according to the Edinburgh handedness inventory (Oldfield, 1971) and were naive as to the purpose of the experiment. The experimental session lasted for approximately $60 \mathrm{~min}$.

Apparatus and Materials. The experiment was conducted in a dark room. Tactile and visual stimuli were generated and the experiment was controlled by MATLAB (MathWorks) with the Psycho- physics Toolbox extension (Brainard, 1997; Pelli, 1997) on a computer (Apple, Power Macintosh G4). The visual stimuli (visual distractor stimuli, picture of a hand, and fixation point) were presented on a display (NEC, CDT1772A) positioned in front of the participants.

Tactile target stimuli were presented via two vibrators (Syainidensi, Attachable Speaker, M-PZT03) attached to a thin-styrofoam box $(17.5 \times 7.5 \mathrm{~cm})$ on a table (Figure 1$)$. The box was filled with sponge to prevent vibrations being transmitted to the whole of the box. The positions of the two vibrators were adjusted to place them under the tip and base of the participant's left forefinger. The tactile target stimulus consisted of three suprathreshold vibration bursts of $200 \mathrm{~Hz}$ (three successive 50-msec bursts separated by 50 -msec intervals). A square with sides of $2 \mathrm{~mm}$ was presented on the display at eye level and served as the fixation point. The visual distractor was presented from two circles of 5-mm diameter positioned $1 \mathrm{~cm}$ above and below the fixation point. The visual distractor stimulus consisted of three yellow flashes of the circle of 50-msec duration each, separated by $50 \mathrm{msec}$ (i.e., the same time course as the tactile target). The onset of the visual distractor was $30 \mathrm{msec}$

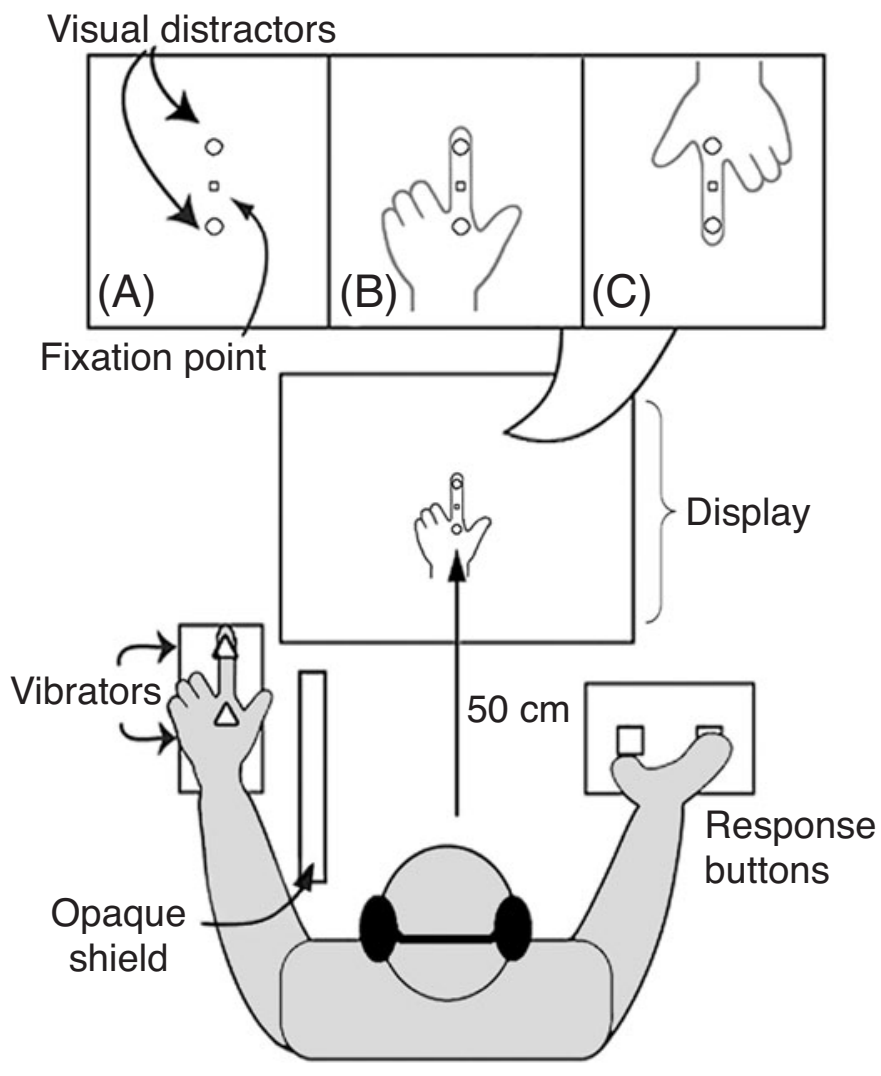

Figure 1. Schematic illustration of the set-up used in Experiment 1. Two vibrators were attached to a styrofoam box. They were positioned under the tip and base of the participant's left hand. Visual distractors were presented from two circles on the display. An opaque shield prevented the participant from seeing the left hand directly. Two response buttons were positioned horizontally under the participant's right hand. Three panels in the rectangular balloon show the visual stimuli that were presented in the three different conditions. (A) In the no-picture condition, only the fixation point and the visual distractor circles were presented on the display. (B) In the aligned picture condition, the hand picture pointing upward was superimposed on the visual distractor circles. (C) In the inverted picture condition, the hand picture was presented pointing downward instead. 
earlier than that of the tactile target, to maximize cross-modal congruency effects (cf. Spence et al., 2004). The picture of a left hand $(6 \times 4.5 \mathrm{~cm})$ with an extended forefinger was drawn in black outline on a white background. The two circles that provided the visual distractors were positioned at the tip and base of the forefinger in the picture. The positions of the visual distractors relative to the picture of the hand corresponded to the positions of the two vibrators relative to the participant's left hand (see Figure 1).

Procedure. The participants sat in front of the display at a distance of $50 \mathrm{~cm}$, with the head fixed on a chinrest. They were instructed to maintain their eyes on the fixation point throughout the trials. They rested their left hands on the table and held the box on which the vibrators were mounted in a forward-pointing posture (see Figure 1). An opaque shield prevented the participants from seeing their left hands and the box held in the participants' hands. On each trial, the tactile target stimulus was presented from one of the two vibrators at the tip or the base of the participant's forefinger, and the visual distractor stimulus was presented from one of the two circles on the display. The positions of the tactile target and the visual distractor were randomly and independently determined on each trial. The participants were instructed to indicate whether the tactile target was presented from either the tip or the base of the forefinger of their left hand by pressing either the left or the right button. Half of the participants responded to the target at the tip with the left button and the target at the base with the right button (i.e., up-left/down-right S-R mapping), and the other half of the participants responded vice versa (i.e., up-right/down-left $S-R$ mapping). They were instructed to respond as rapidly and accurately as possible and to ignore the visual stimuli (both the visual distractors and the hand picture) as much as possible. White noise was presented over headphones to mask any potential auditory cues emitted by the tactile target vibrators.

In the aligned picture condition, the participants performed the tactile discrimination task with the hand picture pointing upward superimposed on the visual distractor circles (see Figure 1). In the inverted picture condition, the hand picture was rotated $180^{\circ}$ (i.e., inverse orientation relative to the participant's left hand). In the no-picture condition, only the fixation point and the visual distractors were presented, and the participants performed the tactile discrimination task without any picture of the hand being presented. The three different picture conditions were presented in separate blocks. The order of the three blocks was randomized between participants. Each block consisted of 20 practice trials (which were not analyzed), followed by 200 test trials (the top or bottom visual distractors $\times$ the tactile target at the tip or base $\times 50$ trials). On the practice trials only, feedback for the responses was presented by changing the color of the fixation point. That is, the color of the fixation point turned green for $1 \mathrm{sec}$ following a correct response and turned red following an incorrect response. The hand picture and the visual distractor circles remained displayed throughout the experimental block. Before the three cross-modal blocks (the no-picture, aligned picture, and inverted picture conditions), the participants performed a unimodal session (20 practice trials and 100 test trials) in which just the fixation point was presented on the display, to get used to the tactile discrimination task (this session was not analyzed).

\section{Results and Discussion}

We discarded recursively those trials in which the reaction time (RT) exceeded \pm 3 standard deviations from the mean RT for each participant. Through this manipulation, $3.1 \%$ of the trials were removed. Trials in which the participants made an incorrect response were also discarded from RT analysis. We modified the manner in which the cross-modal congruency effect had typically been calculated in previous studies (e.g., Maravita et al.,
2003; Spence et al., 2004). For each tactile position (the tip and base) of the finger and for each hand picture condition, we subtracted the mean RTs and error rates for the bottom visual distractor trials from the mean RTs and error rates for the top visual distractor trials (the crossmodal congruency effect) in order to compare the effects of the top and the bottom visual distractors on the tactile discrimination performance. Positive cross-modal congruency effects therefore indicate that the tactile discrimination performance was slower and less accurate with the top visual distractor than with the bottom visual distractor. Negative cross-modal congruency effects indicate that the tactile discriminations were slower and less accurate with the bottom visual distractor than with the top visual distractor. The average mean RTs, error rates, and the cross-modal congruency effects for each condition are shown in Table 1. The RT and error data were analyzed by means of a three-way analysis of variance (ANOVA), with two within-subjects factors of tactile target position (tip vs. base) and hand picture condition (no, aligned, and inverted picture) and a between-subjects factor of $S$ - $R$ mapping (up-right/down-left vs. up-left/ down-right).

The analysis of the RT data showed a significant main effect of tactile target position $[F(1,30)=309.15, p<$ $.001]$, as did the analysis of the error data $[F(1,30)=$ 79.43, $p<.001]$. In all three hand picture conditions, we found that the tactile discrimination responses at the tip were slower and less accurate when the bottom visual distractors were presented (i.e., the negative cross-modal congruency effect), whereas the tactile discrimination responses at the base were slower and less accurate when the top visual distractors were presented (i.e., the positive cross-modal congruency effect; see Table 1 and Figure 2). These results are consistent with previous studies (see, e.g., Maravita et al., 2003, for a recent review) that have repeatedly shown that both the speed and the accuracy of tactile discrimination performance are worse when the positions of tactile targets and visual distractors are spatially incongruent than when they are spatially congruent.

The particular interest of the present study was whether the cross-modal congruency effects would be modulated by the presence of the hand picture and/or by its orientation. The magnitude of the cross-modal congruency effects observed in the no-picture and the aligned picture conditions was indistinguishable, indicating that the presence of the hand picture did not enhance the effect of visual distractors on the tactile discrimination performance (see Figure 2). The magnitude of the cross-modal congruency effects for RT in the inverted picture condition was smaller than those in the aligned picture and no-picture conditions, resulting in the borderline significant interaction between tactile target position and hand picture condition for the RT data $[F(2,30)=2.96, p=$ $.067]$, but not for the error data $[F(2,30)<1]$. There were no significant modulatory effects of the presence and orientation of the hand picture on the cross-modal con- 
Table 1

Mean Reaction Times (RTs, in Milliseconds), Percentages of Errors, and Mean Cross-Modal Congruency Effects as a Function of Hand Picture Condition, Tactile Target Position, and Visual Distractor Position

\begin{tabular}{|c|c|c|c|c|c|c|c|c|c|c|c|c|c|c|c|c|c|}
\hline \multirow{4}{*}{$\begin{array}{l}\text { Tactile } \\
\text { Target } \\
\text { Position }\end{array}$} & \multirow{4}{*}{$\begin{array}{l}\text { Visual } \\
\text { Distractor } \\
\text { Position }\end{array}$} & \multicolumn{16}{|c|}{ Condition } \\
\hline & & \multicolumn{4}{|c|}{ Unimodal } & \multicolumn{4}{|c|}{ No Picture } & \multicolumn{4}{|c|}{ Aligned Picture } & \multicolumn{4}{|c|}{ Inverted Picture } \\
\hline & & \multicolumn{2}{|c|}{$\mathrm{RT}$} & \multicolumn{2}{|c|}{$\%$ Error } & \multicolumn{2}{|c|}{ RT } & \multicolumn{2}{|c|}{$\%$ Error } & \multicolumn{2}{|c|}{ RT } & \multicolumn{2}{|c|}{$\%$ Error } & \multicolumn{2}{|c|}{ RT } & \multicolumn{2}{|c|}{ \% Error } \\
\hline & & $M$ & $\overline{S E}$ & $\bar{M}$ & $\overline{S E}$ & $M$ & $\overline{S E}$ & $M$ & $\overline{S E}$ & $M$ & $\overline{S E}$ & $M$ & $\overline{S E}$ & $M$ & $\overline{S E}$ & $M$ & $S E$ \\
\hline \multirow[t]{3}{*}{ Tip } & Top & \multirow{3}{*}{497} & \multirow{3}{*}{24} & \multirow{3}{*}{3} & \multirow{3}{*}{1} & 516 & 29 & 2 & 1 & 513 & 17 & 2 & 1 & 501 & 22 & 3 & 1 \\
\hline & Bottom & & & & & 612 & 36 & 15 & 4 & 613 & 17 & 18 & 6 & 561 & 22 & 14 & 4 \\
\hline & Mean cros & & & & & -96 & & -13 & & -101 & & -16 & & -60 & & -11 & \\
\hline \multirow[t]{3}{*}{ Base } & Top & \multirow{3}{*}{510} & \multirow{3}{*}{28} & \multirow{3}{*}{$\begin{array}{c}3 \\
\text { ency }\end{array}$} & \multirow{3}{*}{1} & 619 & 36 & 15 & 3 & 601 & 21 & 10 & 3 & 587 & 30 & 13 & 3 \\
\hline & Bottom & & & & & 527 & 34 & 3 & 1 & 510 & 19 & 3 & 1 & 508 & 21 & 3 & 1 \\
\hline & Mean cross-modal congruency effect & & & & & 92 & & 12 & & 91 & & 7 & & 79 & & 10 & \\
\hline
\end{tabular}

Note-The cross-modal congruency effects were calculated by subtracting mean RTs and error rates for the bottom visual distractor trials from those for the top visual distractor trials.

gruency effects. However, the possibility remains that the cross-modal congruency effects might be robust enough to be simply unaffected by the presence of the picture of the hand. The locations of the tactile targets and the visual distractors were aligned vertically and, therefore, were compatible with each other in terms of their spatial alignment. We thought it possible that this high spatial compatibility between them might be too strong to be influenced by the presence and/or orientation of the hand picture. Certainly, the cross-modal congruency effects in the no-picture condition were approximately $100 \mathrm{msec}$ in magnitude (see Table 1), very similar to those that have been reported previously (cross-modal congruency effects are generally reported to be less than 100 msec; Maravita, Spence, Kennett, \& Driver, 2002; Maravita, Spence, Sergent, \& Driver, 2002; Pavani \& Castiello, 2004; Pavani et al., 2000; Spence et al., 2004). The fact that the magnitude of the cross-modal congruency effects did not increase in the aligned picture condition could be due to a ceiling effect. Furthermore, the borderline significant interaction between target position and hand picture condition might reflect the modulatory effect of the inverse orientation of the hand picture. ${ }^{1}$

In this experiment, the orientation of the response button array was orthogonal to the tactile target and visual distractor arrays. The orthogonal S-R compatibility effects

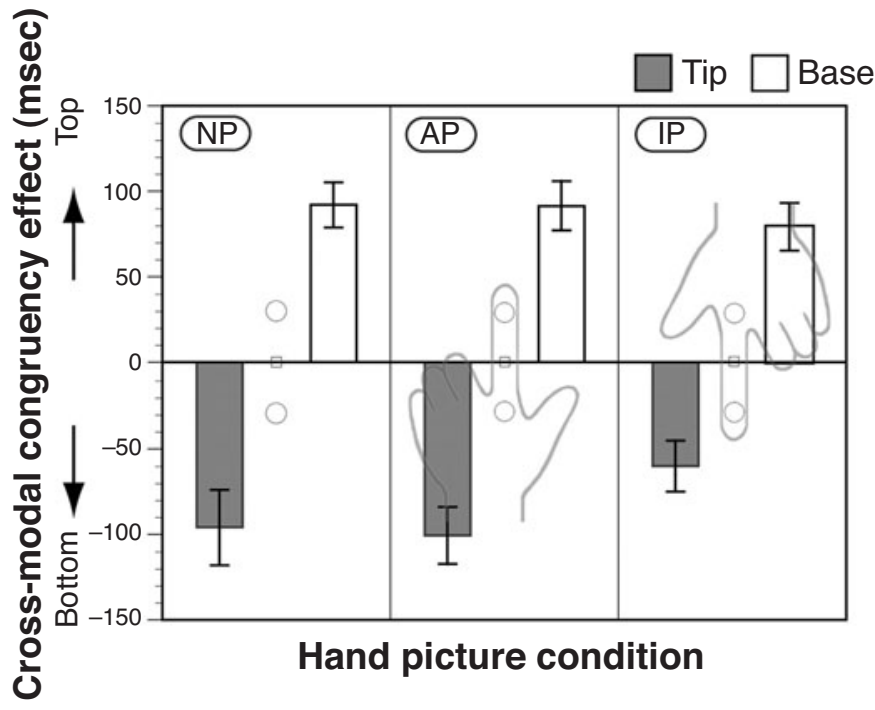

Figure 2. Mean cross-modal congruency effects in the reaction time (RT) data (i.e., RT for the top visual distractor trials minus RT for the bottom visual distractor trials) in Experiment 1 as a function of tactile target position and hand picture condition (NP, no-picture condition; AP, aligned picture condition; IP, inverted picture condition). The positive (negative) values of the cross-modal congruency effect indicate that the tactile discrimination responses were slower when the top (bottom) visual distractors were presented than when the bottom (top) visual distractors were presented. Error bars indicate the standard errors of the means. 
for the tactile target and response arrays were observed as has been summarized by Cho and Proctor (2003). Tactile discriminations were faster when the mapping between the tactile targets and the response buttons was up-right/ down-left $(M=529 \mathrm{msec})$ than when it was up-left/ down-right $(M=583 \mathrm{msec})$. Moreover, strong S-R compatibility effects were also observed between the visual distractor and the response arrays (see Figure 3). The analysis revealed a significant interaction between tactile target position and $\mathrm{S}-\mathrm{R}$ mapping $[F(1,30)=33.25$, $p<.001]$, caused by the larger magnitude of the crossmodal congruency effect with the up-left/down-right mapping than with the up-right/down-left mapping. When the S-R mapping between the tactile target and the response arrays was less compatible (i.e., up-left/ down-right mapping), the visual distractors disrupted the tactile discriminations to a greater extent, presumably because the visual distractors primed responses that were mapped to the response array in a more compatible fashion (i.e., top-right/bottom-left mapping). The results suggest that $\mathrm{S}-\mathrm{R}$ compatibility has considerable effects on human performance not only between responded stimuli and responses, but also between ignoring taskirrelevant stimuli and responses.

In Experiment 1, we observed no significant modulatory effects of the picture of the hand on cross-modal congruency effects. However, given the possibility that the spatial compatibility between the tactile targets and the visual distractors was too strong to be modulated by the picture of the hand, we changed the spatial configuration between them in our second experiment so that they were now positioned orthogonally (hence, reducing the spatial compatibility between the tactile targets and the visual distractors). We predicted that the magnitude of the crossmodal congruency effects should be reduced, as compared with that in Experiment 1, and that the presence and/or orientation of the hand picture would modulate the magnitude of the cross-modal congruency effect more than in our first experiment.

\section{EXPERIMENT 2}

In Experiment 2, we rotated the visual distractors and the hand picture $\pm 90^{\circ}$ from their orientation in Experiment 1 , to misalign the visual distractors with the tactile targets (i.e., the orientation of the visual distractors was orthogonal to that of the tactile targets). We conducted two separate experimental sessions for each of the left and the right hands. In the left-hand session, the tactile targets were presented at the tip and base of the participant's left hand, and the participant responded to the tactile targets with the right hand. In the right-hand session, the role of the hands was reversed - that is, the participant responded with the left hand to the tactile targets presented to the right hand. The hand picture was of either a left or a right hand (i.e., the picture of the left hand for the left stimulated hand, and the picture of the right hand for the right stimulated hand).

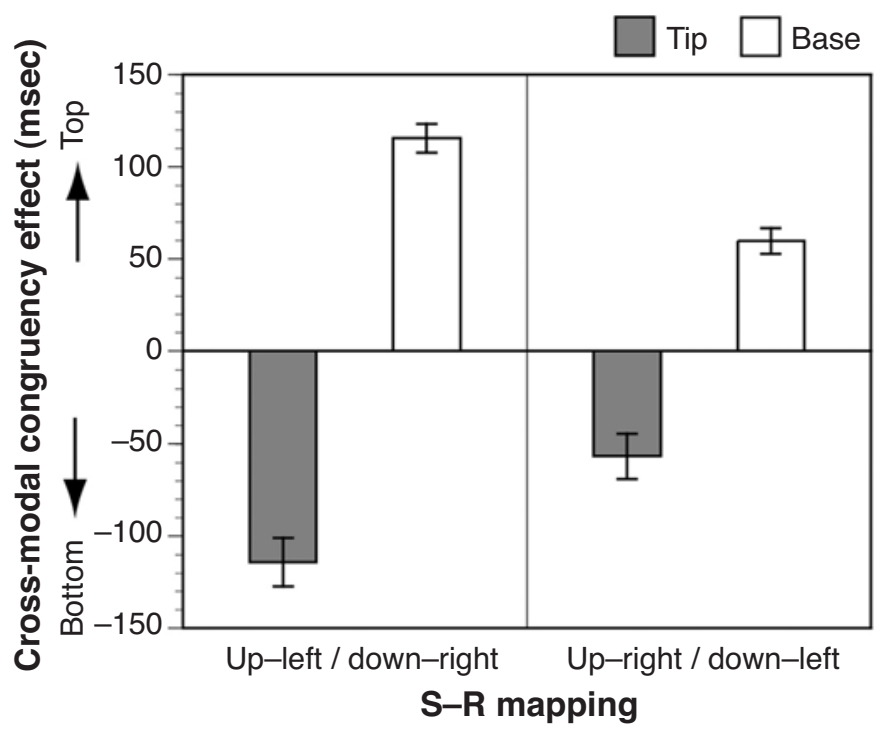

Figure 3. Mean cross-modal congruency effects in the reaction time (RT) data (i.e., RT for the top visual distractor trials minus RT for the bottom visual distractor trials) in Experiment 1 as a function of tactile target position and stimulus-response ( $S-R)$ mapping between the tactile target positions and the response button positions. The positive (negative) values of the cross-modal congruency effect indicate that the tactile discrimination responses were slower when the top (bottom) visual distractors were presented than when the bottom (top) visual distractors were presented. Error bars indicate the standard errors of the means. 


\section{Method}

Participants. Twelve participants ( 3 males and 9 females; mean age, 22.3 years; range, 20-30 years) participated in the left-hand session, and 12 participants (4 males and 8 females; mean age, 20.8 years; range, 19-23 years) participated in the right-hand session. Two of the participants took part in both sessions. All the participants reported having normal touch and normal or corrected-tonormal vision. All were right-handed according to the Edinburgh handedness inventory (Oldfield, 1971).

Apparatus, Materials, and Procedure. The apparatus, materials, and procedure were the same as those in Experiment 1, except for the orientation of the visual stimuli and the response buttons (see Figure 4). The visual distractors were now presented from two circles positioned horizontally, orthogonal to the tactile targets. The two response buttons were placed vertically, orthogonal to the orientation of the visual distractor circles, and were aligned with the tactile targets. In the no-picture condition, the participants performed the tactile discrimination task with the fixation point and the two visual distractor circles presented, but without the hand picture. In the leftward picture condition, the hand picture was superimposed on the visual distractor circles so that the forefinger of the hand picture pointed to the left. In the rightward picture condition, the hand picture was rotated $180^{\circ}$, and its forefinger pointed to the right. All of the participants responded to the tip targets with the top button and to the base targets with the bottom button.

\section{Results and Discussion}

The data were analyzed in the same manner as that in Experiment 1. We discarded recursively the trials in which the RT exceeded \pm 3 standard deviations from the mean RT for each participant. Through this manipulation, $3.8 \%$ of trials in the left-hand session and $3.7 \%$ of trials in the right-hand session were removed. Trials with an incorrect response were also discarded from the RT analysis. For each tactile target position, we subtracted the mean RTs and error rates for the left visual distractor trials from those for the right visual distractor trials (the cross-modal congruency effect) in order to compare the effects of the left and the right visual distractors on the tactile discrimination at each stimulated position. Thus, the positive (negative) values of the cross-modal

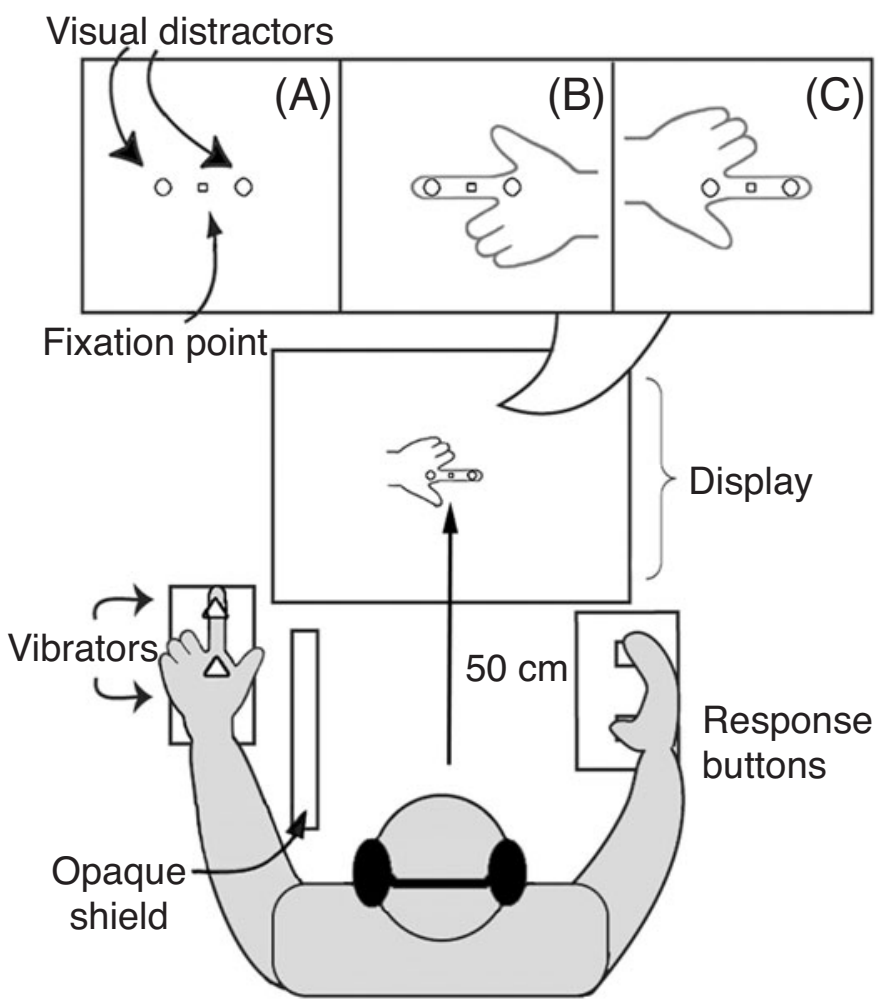

Figure 4. Schematic illustration of the set-up used in the left-hand session of Experiment 2. The visual distractor circles were presented horizontally. Two response buttons were positioned vertically under the participant's right hand. Three panels in the rectangular balloon show the visual stimuli presented in the three different conditions. (A) In the no-picture condition, only the fixation point and the visual distractor circles were presented. (B) In the leftward picture condition, the hand picture pointing leftward was superimposed on the visual distractor circles. (C) In the rightward picture condition, the hand picture pointing rightward was presented. In the right-hand session, the arrangement of the apparatus and the hand picture were reversed for the participant's right hand. 
congruency effect indicate that the tactile discrimination performance was slower and less accurate with the right (left) visual distractors than with the left (right) visual distractors. Table 2 shows the average mean RTs, error rates, and the cross-modal congruency effect for each condition, along with the left- and right-hand sessions. The first thing to note is that the magnitudes of the crossmodal congruency effects were generally smaller than those observed in Experiment 1 (compare Tables 1 and 2). As was expected, the orthogonal layout of the tactile targets and visual distractors presumably weakened the linkage between them, and so the cross-modal congruency effects were consequently reduced. The data from the left- and right-hand sessions were analyzed separately by means of a two-way ANOVA, with within-subjects factors of tactile target position (tip vs. base) and hand picture condition (no, leftward, and rightward picture).

The critical finding was that the orientation of the hand picture modulated the cross-modal congruency effects. The visual distractors that disrupted the tactile discrimination at the tip or the base switched their positions with each other along with the orientation of the hand picture. For both the left- and right-hand sessions (see left panel and right panel of Figure 5, respectively), in the leftward picture condition, the cross-modal congruency effect at the tip had a positive value, whereas the cross-modal congruency effect at the base had a negative value. On the other hand, the opposite trends were obtained in the rightward picture condition - that is, the tactile discrimination responses at the tip were slower when accompanied by the left visual distractors, and the tactile discrimination responses at the base were slower when accompanied by the right visual distractors.

The analysis of the left-hand session showed a significant interaction between the tactile target position and the hand picture condition for both the RT data $[F(2,22)=$ $16.88, p<.01]$ and the error data $[F(2,22)=36.00, p<$
.01]. Post hoc pairwise comparisons (Tukey's HSD; $p<$ .05 ) showed that the cross-modal congruency effect at the tip in the leftward picture condition differed significantly from those in the rightward picture and the no-picture conditions, and similarly at the base, there were significant differences of the cross-modal congruency effects between the leftward picture condition and the other two conditions. The analysis of the right-hand session also showed a significant interaction for the RT data $[F(2,22)=10.77$, $p<.01]$, but not for the error data $[F(2,22)=2.10$, n.s. $]$. The post hoc pairwise comparison for the RT data also showed that the cross-modal congruency effect at the base in the leftward picture condition differed significantly from those in the other two conditions and that the cross-modal congruency effect at the tip in the leftward picture condition differed significantly from that in the rightward picture condition. The results revealed that when the visual distractor positions relative to the hand picture were incongruent with the tactile target positions (i.e., the tactile target at the tip with the visual distractor at the base of the hand picture and the tactile target at the base with the visual distractor at the tip of the hand picture), the tactile discrimination performance was worse than when they were congruent. The finding that the direction of the cross-modal congruency effect changed in accordance with the orientation of the hand picture suggests that the tip and the base of the hand picture corresponded to those of the participant's own hand.

In the no-picture condition, opposite trends for the cross-modal congruency effects between the left- and the right-hand sessions were obtained, although there were no specific spatial correspondences between the tactile targets and the visual distractors under these conditions (i.e., see the top row of Figure 5). The cross-modal congruency effect was negative at the tip and positive at the base in the left-hand session, whereas this was reversed in the right-hand session. The results suggest that the visual

Table 2

Mean Reaction Times (RTs, in Milliseconds), Percentages of Errors, and Mean Cross-Modal Congruency Effects as a Function of Hand Picture Condition, Tactile Target Position, and Visual Distractor Position, Along With the Left- and Right-Hand Sessions

\begin{tabular}{|c|c|c|c|c|c|c|c|c|c|c|c|c|c|c|c|c|c|c|}
\hline \multirow[b]{4}{*}{ Session } & \multirow{4}{*}{$\begin{array}{c}\text { Tactile } \\
\text { Target } \\
\text { Position }\end{array}$} & \multirow{4}{*}{$\begin{array}{c}\text { Visual } \\
\text { Distractor } \\
\text { Position }\end{array}$} & \multicolumn{16}{|c|}{ Condition } \\
\hline & & & \multicolumn{4}{|c|}{ Unimodal } & \multicolumn{4}{|c|}{ No Picture } & \multicolumn{4}{|c|}{ Leftward Picture } & \multicolumn{4}{|c|}{ Rightward Picture } \\
\hline & & & \multicolumn{2}{|c|}{ RT } & \multicolumn{2}{|c|}{$\%$ Error } & \multicolumn{2}{|c|}{ RT } & \multicolumn{2}{|c|}{$\%$ Error } & \multicolumn{2}{|c|}{ RT } & \multicolumn{2}{|c|}{$\%$ Error } & \multicolumn{2}{|c|}{ RT } & \multicolumn{2}{|c|}{$\%$ Error } \\
\hline & & & $M$ & $S E$ & $M$ & $S E$ & $M$ & $S E$ & $M$ & $S E$ & $M$ & $S E$ & $M$ & $S E$ & $M$ & $S E$ & $M$ & $S E$ \\
\hline \multirow[t]{6}{*}{ Left hand } & \multirow[t]{3}{*}{ Tip } & Right & \multirow{3}{*}{447} & \multirow{3}{*}{18} & \multirow{3}{*}{$\begin{array}{c}1 \\
\text { uen }\end{array}$} & \multirow{3}{*}{0} & 459 & 17 & 6 & 2 & 506 & 23 & 11 & 2 & 479 & 18 & 3 & 1 \\
\hline & & Left & & & & & 483 & 19 & 10 & 3 & 492 & 19 & 4 & 2 & 510 & 23 & 12 & 3 \\
\hline & & Mean cro & & & & & -24 & & -4 & & 14 & & 7 & & -31 & & -9 & \\
\hline & \multirow[t]{3}{*}{ Base } & Right & \multirow{3}{*}{442} & \multirow{3}{*}{$\begin{array}{l}15 \\
\text { con }\end{array}$} & \multirow{3}{*}{2} & \multirow{3}{*}{$\begin{array}{l}1 \\
\text { effect }\end{array}$} & 501 & 20 & 6 & 1 & 496 & 18 & 3 & 1 & 529 & 25 & 10 & 3 \\
\hline & & Left & & & & & 468 & 20 & 3 & 1 & 510 & 24 & 6 & 2 & 484 & 22 & 3 & 1 \\
\hline & & Mean cro & & & & & 33 & & 3 & & -13 & & -3 & & 45 & & 6 & \\
\hline \multirow[t]{6}{*}{ Right hand } & \multirow[t]{3}{*}{ Tip } & Right & \multirow{3}{*}{456} & \multirow{3}{*}{19} & \multirow{3}{*}{2} & & 472 & 20 & 7 & 2 & 508 & 22 & 6 & 1 & 469 & 21 & 4 & 1 \\
\hline & & Left & & & & 1 & 460 & 21 & 4 & 1 & 470 & 17 & 5 & 1 & 474 & 25 & 5 & 1 \\
\hline & & Mean cro & & & & effect & 12 & & 3 & & 38 & & 1 & & -5 & & -1 & \\
\hline & Base & Right & 437 & & & & 461 & 21 & 5 & 2 & 461 & 18 & 3 & 1 & 483 & 30 & 5 & 2 \\
\hline & & Left & 431 & 21 & 1 & 1 & 468 & 20 & 4 & 1 & 498 & 22 & 6 & 2 & 472 & 23 & 3 & 1 \\
\hline & & Mean cro & $s-\bmod$ & con & uen & effect & -7 & & 0 & & -37 & & -3 & & 11 & & 2 & \\
\hline
\end{tabular}

Note-The cross-modal congruency effects were calculated by subtracting mean RTs and error rates for the left visual distractor trials from those for the right visual distractor trials. 


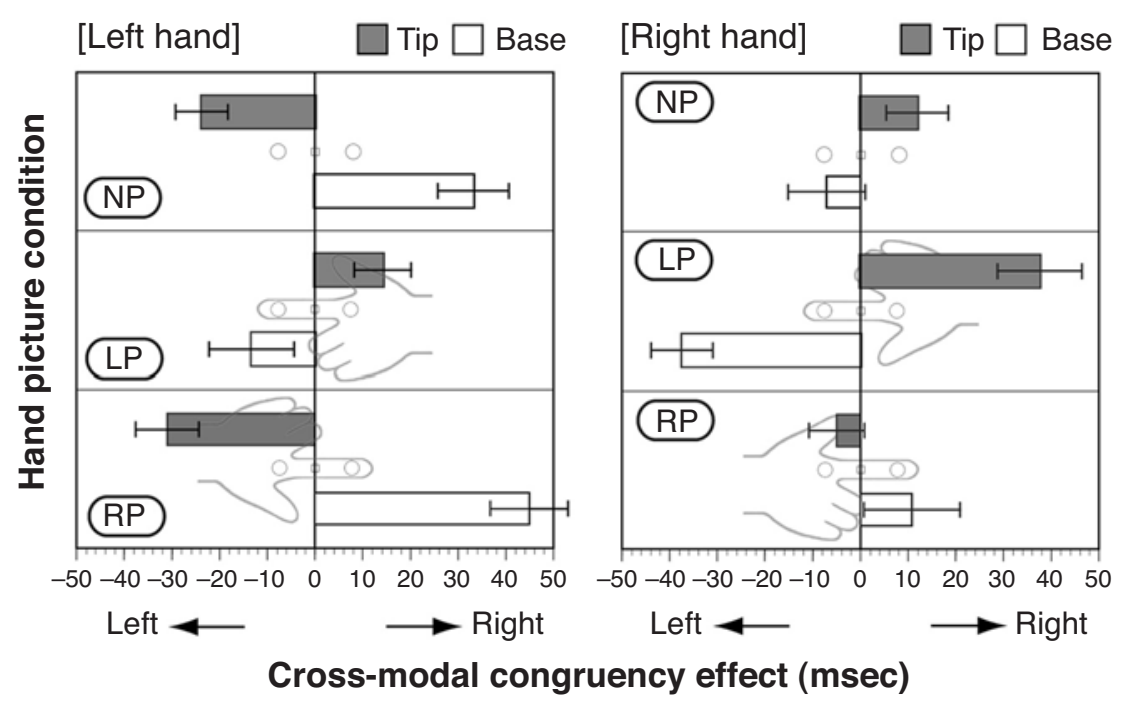

Figure 5. Mean cross-modal congruency effects in the reaction time (RT) data (i.e., RT on the right visual distractor trials minus RT on the left visual distractor trials) in Experiment 2, as a function of tactile target position and hand picture condition (NP, no-picture condition; LP, leftward picture condition; RP, rightward picture condition). The left panel shows the results of the left-hand session, and the right panel shows the results of the right-hand session. The positive (negative) values of the cross-modal congruency effect indicate that the tactile discrimination responses were slower when the right (left) visual distractors were presented than when the left (right) visual distractors were presented. Error bars indicate the standard errors of the means.

distractors influenced the tactile performance as if their positions corresponded to the inward orientation of the participant's own hand. This seems to be plausible, since it was difficult for participants to position their own hands in an outward posture. The same could be argued for the leftward picture and rightward picture conditions - that is, the cross-modal congruency effects were larger when the orientation of the hand picture was congruent with the natural inward posture of the responding hand (i.e., the rightward picture condition of the left-hand session and the leftward picture condition of the right-hand session) than when they were incongruent (i.e., the leftward picture condition of the left-hand session and the rightward picture condition of the right-hand session).

Similar opposite trends have been reported for the orthogonal S-R compatibility effects when stimuli are presented in a plane orthogonal to the response array (Bauer \& Miller, 1982; Lippa, 1996; Lippa \& Adam, 2001). For this type of orthogonal S-R compatibility effect, the mapping preference (i.e., up-left/down-right vs. up-right/ down-left) varies with the responding hand, its posture, and the spatial relationship between the stimulus and the response arrays. Lippa and Adam assumed that when the stimulus and the response arrays are orthogonal, we mentally rotate the response array to match the orientation of the stimulus array, and thus, the mapping preference depends on the direction that yields the most comfortable posture of the responding hand-the so-called end-state comfort hypothesis. The similarity between the orthogo- nal S-R compatibility effects and the cross-modal congruency effects observed here suggest that the spatial linkage between stimulus and response arrays examined in terms of the S-R compatibility is not limited to the spatial relationship between them but that the linkage may actually exist in the spatial relationship between the stimulus array and one's own hand.

\section{GENERAL DISCUSSION}

In the present study, we investigated whether a simple drawing of a hand would modulate interactions between vision and touch, as has been reported for projected images of our own body parts in a mirror reflection (Maravita, Spence, Sergent, \& Driver, 2002; Soto-Faraco et al., in press), in a video monitor (Tipper et al., 1998; Tipper et al., 2001), and as a cast shadow (Pavani \& Castiello, 2004). To this end, we examined whether the presence and orientation of a simple picture of a hand modulated visual-tactile cross-modal congruency effects. Our results revealed that the picture of the hand and the posture of the stimulated and responding hand significantly influenced the tactile discrimination performance.

First, the hand picture modulated visual-tactile interactions. The effects of the hand picture were small when the visual distractors were spatially aligned with the tactile targets in Experiment 1-that is, a small (and nonsignificant) reduction in magnitude of the cross-modal congruency effects was observed when the hand picture 
was presented in the inverse orientation, as compared with the participant's own hand. However, the effects of the hand picture were much larger when the visual distractors were spatially misaligned with the tactile targets in Experiment 2. The direction of the cross-modal congruency effects (i.e., which visual distractor led to slower and less accurate tactile discrimination at each stimulated position) was changed along with the orientation of the hand picture, as if the hand picture corresponded to the participant's own hand. These results suggest that even a two-dimensional drawing of a hand can modulate the processes maintaining our internal body representation.

Second, the magnitude of the cross-modal congruency effect was approximately $100 \mathrm{msec}$ when the visual distractors were spatially aligned with the tactile targets (Experiment 1). On the other hand, when the visual distractor and the tactile target arrays were orthogonal to each other (Experiment 2), the cross-modal congruency effects were weak - that is, even the maximum mean cross-modal congruency effect in Experiment 2 was less than half that in Experiment 1. These results indicate that the spatial correspondence between visual and tactile stimuli is a much more important factor for visualtactile spatial interactions than the modulatory effect of the picture of the hand.

Third, the direction and magnitude of the cross-modal congruency effects also depended on the posture of the participants' stimulated hand. When the participants performed the cross-modal congruency task without the hand picture in the no-picture condition of Experiment 2, the direction of the cross-modal congruency effects depended on the most comfortable posture (i.e., the inward posture) of the stimulated hand. The effects of the posture of the stimulated hand were also observed in the other conditions in which the hand picture was presented. When the orientation of the hand picture and the inward posture of the stimulated hand were congruent, larger crossmodal congruency effects were obtained, but when they were incongruent, the magnitudes of the cross-modal congruency effects were smaller. For this point, the result of Experiment 1 that the inverse orientation of the hand picture did not have a significant influence upon the cross-modal congruency effects might be explained by the fact that the inverse posture was anatomically impossible for the participant's stimulated hand.

Fourth, S-R compatibility effects were obtained not only for the spatial configuration of tactile target and response arrays but also for the configuration between the visual distractor and the response arrays. In Experiment 1, the tactile targets and the visual distractors were presented vertically, whereas the response buttons were positioned horizontally (i.e., orthogonal configuration). Tactile discrimination performance was better when the tactile targets were mapped to the response buttons in the up-right/down-left configuration than when they were mapped in the up-left/down-right configuration. Furthermore, the cross-modal congruency effects with the nonpreferred mapping (i.e., up-left/down-right) were stronger than those with the preferred mapping (i.e., up-right/down-left; see Figure 3), presumably because the visual distractors primed the responses in accordance with the preferred up-right/down-left mapping between the visual distractor and the response arrays. These results suggest that $\mathrm{S}-\mathrm{R}$ compatibility is an important factor for human performance even when stimuli are irrelevant to a given task and they are ignored.

It has been reported that the active wielding of a long tool affects visual-tactile interactions as if the tip of the tool is remapped as the extension of our own hands (Berti \& Frassinetti, 2000; Farnè \& Làdavas, 2000; Maravita, Spence, Kennett, \& Driver, 2002). This possible alteration of the body representation by tool use has been extended to the situation in which a tool and a hand were presented in a video monitor. After monkeys were trained to use a tool projected in a video monitor, the bimodal neurons that usually respond to tactile stimuli on the hands and visual stimuli just around the hands also began to respond to visual stimuli around the end of the tool shown in the monitor (Iriki, Tanaka, Obayashi, \& Iwamura, 2001). Given these results and the results obtained in this study, it would be interesting to examine whether prolonged manipulation of a picture of a hand or even more simple objects would have any effect on visual-tactile interactions.

Finally, in the present study, we reported that the simple line drawing of a hand modulated visual-tactile interactions. However, the possibility remains that the hand picture led the participants to think of that particular body site (the tip and base of the forefinger) and, thereby, modulated the cross-modal congruency effects. If this is the case, the words of the body parts (e.g., fingertip) presented by the visual distractors should have the same modulatory influence on the cross-modal congruency effect. In future research, it would be worth identifying whether the modulatory effects observed here are due to the sight of the hand picture itself or whether the effects are mediated by other cognitive processes.

\section{REFERENCES}

BAuer, D. W., \& Miller, J. (1982). Stimulus-response compatibility and the motor system. Quarterly Journal of Experimental Psychology, 34A, 367-380.

BERTI, A., \& FrASSINETTI, F. (2000). When far becomes near: Remapping of space by tool use. Journal of Cognitive Neuroscience, 12, 415-420.

Botvinick, M., \& CoHEN, J. (1998). Rubber hands "feel" touch that eyes see. Nature, 391, 756 .

Brainard, D. H. (1997). The Psychophysics Toolbox. Spatial Vision, 10, 433-436.

CHO, Y. S., \& Proctor, R. W. (2003). Stimulus and response representations underlying orthogonal stimulus-response compatibility effects. Psychonomic Bulletin \& Review, 10, 45-73.

Di Pellegrino, G., LÀdavas, E., \& FarNè, A. (1997). Seeing where your hands are. Nature, $\mathbf{3 8 8}, 730$.

FARNÈ, A., \& LÀDAVAS, E. (2000). Dynamic size-change of hand peripersonal space following tool use. NeuroReport, 11, 1645-1649.

Farnè, A., Pavani, F., Meneghello, F., \& Làdavas, E. (2000). Left tactile extinction following visual stimulation of a rubber hand. Brain, 123, 2350-2360.

FiTTS, P. M., \& DEININGER, R. L. (1954). S-R compatibility: Correspon- 
dence among paired elements within stimulus and response codes. Journal of Experimental Psychology, 48, 483-492.

Graziano, M. S. A. (1999). Where is my arm? The relative role of vision and proprioception in the neuronal representation of limb position. Proceedings of the National Academy of Sciences, 96, 10418-10421.

Graziano, M. S. A., CoOKe, D. F., \& TAYlor, C. S. R. (2000). Coding the location of the arm by sight. Science, 290, 1782-1786.

Iriki, A., TanaKa, M., ObaYashi, S., \& IWAMURA, Y. (2001). Self-images in the video monitor coded by monkey intraparietal neurons. Neuroscience Research, 40, 163-173.

LÀDAVAS, E. (2002). Functional and dynamic properties of visual peripersonal space. Trends in Cognitive Sciences, 6, 17-22.

LÀdavas, E., Farnè, A., Zeloni, G., \& di Pelle Grino, G. (2000). Seeing or not seeing where your hands are. Experimental Brain Research, 131, 458-467.

LIPPA, Y. (1996). A referential-coding explanation for compatibility effects of physically orthogonal stimulus and response dimensions. Quarterly Journal of Experimental Psychology, 49A, 950-971.

LiPPA, Y., \& ADAM, J. J. (2001). An explanation of orthogonal S-R compatibility effects that vary with hand or response position: The endstate comfort hypothesis. Perception \& Psychophysics, 63, 156-174.

Lloyd, D. M., Shore, D. I., Spence, C., \& Calvert, G. A. (2003). Multisensory representation of limb position in human premotor cortex. Nature Neuroscience, 6, 17-18.

Maravita, A., SPEnCE, C., \& DrIVER, J. (2003). Multisensory integration and the body schema: Close to hand and within reach. Current Biology, 13, R531-R539.

Maravita, A., Spence, C., Kennett, S., \& Driver, J. (2002). Tool-use changes multimodal spatial interactions between vision and touch in normal humans. Cognition, 83, B25-B34.

Maravita, A., Spence, C., Sergent, C., \& Driver, J. (2002). Seeing your own touched hands in a mirror modulates cross-modal interactions. Psychological Science, 13, 350-355.

OLDFIELD, R. C. (1971). The assessment and analysis of handedness: The Edinburgh inventory. Neuropsychologia, 9, 97-113.

Pavani, F., \& Castiello, U. (2004). Binding personal and extrapersonal space through body shadows. Nature Neuroscience, 7, 14-16.

Pavani, F., Spence, C., \& Driver, J. (2000). Visual capture of touch:
Out-of-the-body experiences with rubber gloves. Psychological Science, 11, 353-359.

PeLLI, D. G. (1997). The Video Toolbox software for visual psychophysics: Transforming numbers into movies. Spatial Vision, 10, 437442.

Soto-Faraco, S., Ronald, A., \& Spence, C. (in press). Tactile selective attention and body posture: Assessing the multisensory contribution of vision and proprioception. Perception \& Psychophysics.

Spence, C. Kingstone, A., Shore, D. I., \& Gazzaniga, M. S. (2001). Representation of visuotactile space in the split-brain. Psychological Science, 12, 90-93.

Spence, C., PAVANI, F., \& Driver, J. (2004). Spatial constraints on visualtactile cross-modal distractor congruency effects. Cognitive, Affective, \& Behavioral Neuroscience, 4, 152-173.

Tipper, S. P., Lloyd, D., Shorland, B., Dancer, C., Howard, L. A., \& McGlone, F. (1998). Vision influences tactile perception without proprioceptive orienting. NeuroReport, 9, 1741-1744.

Tipper, S. P., Phillips, N., Dancer, C., Lloyd, D., Howard, L. A., \& McGlone, F. (2001). Vision influences tactile perception at body sites that cannot be viewed directly. Experimental Brain Research, 139, 160-167.

WEEKS, D. J., \& Proctor, R. W. (1990). Salient-features coding in the translation between orthogonal stimulus and response dimensions. Journal of Experimental Psychology: General, 119, 355-366.

\section{NOTE}

1. When the participants experienced the aligned or the inverted picture condition prior to the no-picture condition, their cross-modal congruency effects in the no-picture condition might be modulated by the experience. We examined whether there were any order effects of the hand picture conditions. We conducted a two-way ANOVA with a within-subjects factor of tactile target position (tip vs. base) and a between-subjects factor of relative position of no-picture condition (postaligned picture, postinverted picture, before the other two picture conditions). There were no order effects for RT data $[F(2,15)<1]$ and error data $[F(2,15)=$ 1.05 , n.s.], showing the robust spatial compatibility between the tactile targets and the visual distractors.

(Manuscript received October 15, 2003; revision accepted for publication March 26, 2004.) 